\title{
Gene Signature: A Guideline for Hormonal Therapy in Breast Cancer
}

\author{
Nguyen Minh Nam
}

Department of Biochemistry and Molecular Biology, School of Medicine, Kyung Hee University, Seoul, Republic of Korea

*Corresponding author: Nguyen Minh Nam, Department of Biochemistry and Molecular Biology, School of Medicine, Kyung Hee University, 26 Kyungheedae-ro, Dongdaemun-gu, Seoul 130-701, Republic of Korea, Tel: 82-2-961-0287; Fax: 82-2-959-8168; E-mail: minhnam1984@gmail.com

Received date: July 15, 2015, Accepted date: July 18, 2015, Published date: July 24, 2015

Copyright: (C) 2015 Nam NM. This is an open-access article distributed under the terms of the Creative Commons Attribution License, which permits unrestricted use, distribution, and reproduction in any medium, provided the original author and source are credited.

\section{Editorial}

Breast cancer is the most common leading cause of cancer-related death in women world wide and it is a molecular heterogeneous disease. The heterogeneous expression of hormone receptors such as estrogen receptor (ER), progesterone receptor (PR), and HER2, has been used to divide breast cancer patients into intrinsic subtypes based on the present or absence of these hormone receptors. And it also helps guide judicious treatment decisions in response to either hormonal therrapy, chemotherapy, or radiation therapy. Hormonal therapies such as tamoxifen, aromatase inhibitors or any therapies that target HER2 receptors line like Herceptin, are helpful in hormone receptor-positive breast cancers, but not in patients whose tumors are hormone receptor negative. However, identification of patients who might benefit from these treatments and who are at high risk of reoccurrence and resistance after treatment need to improve.

Studies based on global gene expression analyses have the potential to identify breast cancer gene signatures which can improve diagnosis and treatment accurately by identifying patients who could benefit most from treatment. The power of applying customized microarray technology to predict the prognosis of patients in breast cancer led to the successful development of the U.S. Food and Drug Administration-approved breast cancer prognostic test, such as Prosigna and Mamma Print [1,2]. In addition, Oncotype $\mathrm{X}$ is a genomic breast cancer test widely used to make treatment decisions; and it is also the only test included in the National Comprehensive Cancer Center Network (NCCN) and the American Society of Clinical Oncology ASCO) treatment guideline. These prognostic tests have been shown to successfully predict the likelihood of chemotherapy benefits as well as distant recurrence 10 years after diagnosis in patients with early-stage, node-negative and node-positive ER-positive breast cancer. Furthermore, Mamma Print test can significantly predict cancer patients who are estrogen receptor negative. To contribute to this work, we developed a 92 probe signature, as a predictor in breast cancer late recurrences and to identify subtypes within the lymph node positive and ER positive patients that best reflected the invasive breast cancer biology [3]

Triple negative breast cancer (TNBC) represent as a group of several molecularly and clinically distinct disease subtypes. Although TNBC is highly responsive to chemotherapy compared with hormone receptor positive breast cancer, it has a poor overall prognosis. Breast cancer patients who are ER/PR negative are 5\% to $10 \%$ likely to respond to hormonal therapy [4]. However, there are no predictive biomarkers on response or survival to therapy for these patients. It is essential to investigate the biomaker or gene signature to identify breast cancer patients who benefit not only from chemotherapy and radiation therapy but also from hormonal therapy.

The evolution of breast cancer from early stage to advanced-stage is complex and leads to increased heterogeneity. During this disease evolution, there are genetic alterations, new mutations and consequently gene expression pattern changes. New mutation in genes, such as ERS1 [5], are related to acquired resistance to hormonal therapy and present as a potential targets for future therapies. Biomarker status, such as hormonal receptor, is also changed and this change is associated with a poorer prognosis, particularly in the case of ER expression loss and HER2 expression gain. The changes of these receptor status influences treatment choices due to development of resistance during disease evolution. To this end, there are no validated biomarkers for prediction hormonal therapy resistance. So, it is essential to identify a new biomarker as a new target or new gene signature for prediction of the risk of hormonal therapy resistance.

We are currently focusing on finding a gene signature that would provide a new insight into heterogeneity developed during evolution of breast cancer. There is also a need to identify a gene signature and a new target for the prediction of hormonal therapy resistance and combined therapeutic selection. We are studying on triple-negative breast cancer to develop biomarkers or gene signatures for prediction of patients who could be responsive to chemotherapy. We welcome and encourage scientists all around the world to investigate new biomarkers and/or gene signatures for optional therapy selection to increase the care of people diagnosed with cancer.

\section{References}

1. van 't Veer LJ, Dai H, van de Vijver MJ, He YD, Hart AA, et al. (2002) Gene expression profiling predicts clinical outcome of breast cancer. Nature 415: 530-536.

2. Glas AM, Floore A, Delahaye LJ, Witteveen AT, Pover RC, et al. (2006) Converting a breast cancer microarray signature into a high-throughput diagnostic test. BMC Genomics 7: 278.

3. Akter S, Choi TG1, Nguyen MN1, Matondo A1, Kim JH1, et al. (2015) Prognostic value of a 92-probe signature in breast cancer. Oncotarget 6: 15662-15680.

4. Zhang CC, Shapiro DJ (2000) Activation of the p38 mitogen-activated protein kinase pathway by estrogen or by 4-hydroxytamoxifen is coupled to estrogen receptor-induced apoptosis. J Biol Chem 275: 479-486.

5. Robinson DR, Wu YM, Vats P, Su F, Lonigro RJ, et al. (2013) Activating ESR1 mutations in hormone-resistant metastatic breast cancer. Nat Genet 45: 1446-1451. 